\title{
Random field-based modeling of size effect on the longitudinal tensile strength of clear timber
}

\author{
Alireza F. Moshtaghin ${ }^{1}$, Steffen Franke ${ }^{2}$, Thomas Keller ${ }^{1}$ and Anastasios P. Vassilopoulos ${ }^{1 *}$ \\ ${ }^{1}$ Composite Construction Laboratory (CCLab), Ecole Polytechnique Fédérale de Lausanne (EPFL), \\ Station 16, Bâtiment BP, CH-1015 Lausanne, Switzerland \\ 2 Timber and Composite Construction, Bern University of Applied Sciences, Solothurnstrasse 102, \\ Biel, Switzerland
}

\begin{abstract}
In the current study, a random field-based size effect model has been proposed for the longitudinal tensile strength of clear timber. Since the failure mode is brittle, the problem is basically an extreme value problem of finding the distribution of minima of strength fields for specimens of different volumes. The stochastic response has been evaluated based on the Monte Carlo method along with the weakest link theory. Within the framework of the spectral representation method, the Weibull distribution has been considered as the marginal distribution to generate realizations of the strength as a 3D random field. The squared exponential autocorrelation function has been used for the description of spatial variability. The error resulting from this model, as compared to existing experimental data in the literature, is much lower than that of the classical Weibull law. The results show that when one of the specimen's dimensions decreases to less than 10 times the correlation length of the strength field, the size effect starts to deviate from the classical size effect law. Moreover, a simple analytical approximation, which includes the correlation length as length scale, has been presented that facilitates the application of the proposed model.
\end{abstract}

Keywords: clear timber, size effect, longitudinal tensile strength, random fields

\section{Introduction}

From a structural point of view, wood/timber can be considered as a natural unidirectional fiber composite with highly anisotropic properties. For specific species, geographical location, and local growth conditions, the material properties depend on factors such as age, structural imperfections, location of timber within the tree, and load history and are therefore inherently highly variable. One of the consequences of this variability is the phenomenon known as statistical size effect. When the failure mode is brittle, the mean strength of a specimen with a larger volume is lower than that of a smaller one, which is also the case for any other level of cumulative probability, and this difference increases with the level of variability in material properties. This is normally attributed to the higher probability of critical defects occurring in a larger volume.

The classical Weibull size effect law (CWSEL) [1] is the most common model used in the literature [2] for the description of statistical size effects on timber strength in its brittle failure

\footnotetext{
* Corresponding Author: e-mail: anastasios.vasilopoulos@epfl.ch Tel.: +41 216936393 Fax: +41 216936240.
} 
modes. According to this model, a structural member fails when the stress level reaches the strength at a single material point.

The size effect on timber strength can be treated as a volume effect, for example see [3-6] where such an assumption was adopted for the failure analysis of adhesively-bonded, welded and dovetail timber joints and [7-9] in which the elastoplastic behavior of strand-based wood composites and laminated veneer was studied. The Weibull law was applied only in tensile mode, while plastic behavior was considered only in compressive mode. Alternatively, the size effect can be split into length and cross-sectional effects - see e.g. [10,11] - on the strength prediction of clear timber under bending and [12] in the case of structural lumber.

Nevertheless, research efforts for the quantification of size effect on the strength of clear wood, by conducting pure tensile tests on specimens with different volumes, are very limited in the literature. Dill-Langer et al. [13] conducted longitudinal tensile experiments on specimens made of spruce wood. Two groups of specimens with different volumes were tested and a fiber bundle model was introduced in order to simulate the macroscopic behavior in terms of microscopic damage. Zhu et al. [14] introduced a length-effect parameter to quantify the size effect due to the length change on the longitudinal tensile strength of Japanese larch wood.

In current practice using the CWSEL, a Weibull distribution is fitted to data obtained from experiments on specimens with standardized dimensions. Then, the CWSEL is used to predict the strength of pieces of timber either with higher volumes [2,10,11], like timber beams, or lower volumes [4,7], which are usually small elements considered in finite element analyses of timber structures. However, using experimental data from the literature [13,14], it is shown in the current study that this procedure can result in relative errors in the prediction of the size effect on timber strength as high as $400 \%$. This is attributed to the fact that the spatial correlation in the strength field is neglected in the CWSEL. It is noted that the inaccuracy of the CWSEL, when small volumes of materials are concerned, is already known; however, the issue has been overlooked in the case of timber. Nevertheless, it is important, particularly in the FE content, to extend size effect predictions to small volumes.

As an alternative to the CWSEL, timber strength can be modeled as a random field, considering, in addition to strength variation between different specimens as in conventional statistical analyses, the spatial variation within each specimen in a random manner. There are several methods to generate realizations of a random field in each specimen of which the spectral representation method is the most accurate and efficient [15]. A realization is a randomly generated sample of a random field and describes the spatial variability of the field for that sample in the physical domain considered, e.g. the volume of a specimen. Realizations of the strength field for specimens with different volumes can be used for investigating the size effect. Recently, Arwade et al. [16,17] used the random field approach to characterize the lengthwise spatial variability in the elastic modulus and compressive strength of parallel strand lumbers as 1D random fields. However, there is no study in the literature that has been devoted to the size effect on the longitudinal tensile strength of clear timber, and the spatial correlation in the strength field, i.e. the way in which the strength value at one point influences the strength at the surrounding points, has usually been neglected.

Studying the size effect on the longitudinal tensile strength of clear timber using random fields, is a problem of finding the extreme value statistics. This is because the failure mode is 
brittle, and to investigate the size effect, the distributions of minima of strength fields for specimens of different volumes has to be obtained. The extreme value problem was studied thoroughly by Gumbel in 1958 [18]. Concerning random fields, lots of research on this problem has been conducted in different scientific domains, e.g., by mathematicians [19], and in hydrology [20], climate [21], and cosmology [22]. Useful information on this topic can be found in [23]. Analytical solutions are only available for a few cases [24], and therefore, numerical methods such as simulations [25] or asymptotic approximation methods [24] are usually employed.

This study presents a model for the size effect on timber strength that takes into account the spatial variability in the strength field. The theory of random fields was used to model the random 3D spatial variability of the longitudinal strength. Using the spectral representation scheme, realizations of strength field in each specimen were generated considering the Weibull distribution and squared exponential function for statistical variability and spatial variability, respectively. The stochastic response was obtained via the Monte Carlo method along with the weakest link theory. This procedure was repeated for specimens with different volumes to estimate the effect of size on strength. The current results were compared to experimental data from the literature. An analytical expression able to efficiently approximate the numerical results of the random field modeling has also been introduced in this work.

\section{Classical Weibull size effect law and its limitations}

\section{Weibull size effect theory}

The CWSEL has been widely used to model the size effect on timber strength [2,4-12]. According to this model, the material is considered as a structure made up of linked elements, which fails with the first element failure. The mean strength of a specimen under uniaxial loading, $\bar{\sigma}(V)$, is related to its volume $V$ as:

$$
\bar{\sigma}(V)=\sigma_{0} \Gamma(1+1 / m)\left(\frac{V_{0}}{V}\right)^{1 / m}
$$

where $\sigma_{0}$ and $m$ are the scale and shape parameters of the Weibull distribution, $\Gamma$ is the Gamma function, and $V_{0}$ is a reference volume. Eq. (1) can be plotted as a straight line in a $\log$-log scale. It can be shown that, at any given failure probability level, the strengths of two pieces, $\sigma_{1}$ and $\sigma_{2}$, with volumes $V_{1}$ and $V_{2}$ are related:

$$
\frac{\sigma_{1}}{\sigma_{2}}=\left(\frac{V_{2}}{V_{1}}\right)^{1 / m}
$$

based on the assumption of independent identically distributed random variables [26]. For a numerical representation of the method, the larger volume $V_{2}$ is divided into $n$ segments with volumes equal to $V_{1}$ and a random strength value from the Weibull distribution, corresponding to volume $V_{1}$, is assigned to each segment. The minimum value of the segment strengths is considered as the strength of $V_{2}$ based on the weakest link theory. This simulation is repeated as many times as necessary to collect sufficient data points for estimating the 
statistics of the strength distribution of $V_{2}$. When $V_{2} / V_{1}$ is not an integer, interpolation can be used. Finally, having obtained the strength distributions for both volumes, it is easy to show that their relationship follows Eq. (2). However, due to this independent spatial assignment of the strength to smaller segments in the larger volume, The CWSEL tends to overestimate the effect of size on strength [26]. In reality, there is always a spatial correlation in the strength field that can be considered by using the random field approach. Moreover, Eq. (2) implies that the CWSEL can be scaled arbitrarily; i.e., its form does not change even for very small volumes of materials. Therefore, for very small volumes $(V \rightarrow 0)$ the scale parameter, or equivalently the mean value of the strength distribution, approaches infinity. However, this is not the case in reality, and there is an upper limit for strength as volume decreases.

\section{Modeling size effect on longitudinal tensile strength of clear timber with CWSEL}

In most works related to the size effect on the longitudinal tensile strength of clear wood, tensile tests parallel to the grain are only performed on specimens with a constant volume. This is because testing a set of specimens with a constant volume suffices to determine the unknown parameter of the CWSEL. Thus, by fitting the Weibull distribution to the experimental data, the shape parameter is estimated, and the CWSEL is then used in the related application, such as estimation of a wooden joint capacity [4]. To the authors' knowledge, there are only two works $[13,14]$ in which the effect of volume change on the longitudinal tensile strength of clear timber has been investigated experimentally. The specimen dimensions in these works and the corresponding Weibull parameters are given in Table 1. The dimensions correspond to the middle part of specimens with a constant crosssectional area. In [13], longitudinal tensile tests were conducted on two sets of clear specimens made of spruce wood. The dimensions of the larger specimen are approximately three times those of the smaller specimen. In [14], however, only the length of the specimen has been changed. Nevertheless, in each of these works, the Weibull shape parameters obtained from fitting the experimental results of the smaller and larger specimens exhibit some differences. To examine the size effect within the Weibull theory framework, it is necessary to have a constant shape parameter; therefore, an average value was used in this study for examining the accuracy of the CWSEL; i.e., 9.3 and 4.0 for the experimental results from [13] and [14] respectively. The error introduced in the calculation of mean values due to this averaging, compared to mean values from experimental data, is less than $0.5 \%$ in both cases.

Table 1: Experimental results for longitudinal tensile strength of clear timber.

\begin{tabular}{|l|l|l|l|l|l|}
\hline Researchers & Type of wood & $\begin{array}{l}\text { Specimen } \\
\text { dimensions } \\
\left(\mathrm{mm}^{3}\right)\end{array}$ & $\begin{array}{l}\text { Weibull scale } \\
\text { parameter } \\
(\mathrm{MPa})\end{array}$ & $\begin{array}{l}\text { Weibull shape } \\
\text { parameter }\end{array}$ & $\begin{array}{l}\text { Mean value } \\
\text { strength } \\
(\mathrm{MPa})\end{array}$ \\
\hline $\begin{array}{l}\text { Dill-Langer et al. } \\
{[13]}\end{array}$ & spruce & $2 \times 6 \times 35$ & 146.2 & 8.3 & $138.7 \pm 19.8$ \\
\cline { 3 - 6 } & $6 \times 20 \times 110$ & 134.0 & 10.3 & $127.1 \pm 14.9$ \\
\hline Zhu et al. [14] & Japanese larch & $5 \times 25 \times 30$ & 122.9 & 3.77 & $111.4 \pm 32.9$ \\
\cline { 3 - 6 } & & $5 \times 25 \times 120$ & 114.0 & 4.22 & $103.3 \pm 27.7$ \\
\hline
\end{tabular}


When specimen volumes and Weibull shape parameters for spruce are substituted into Eq. (2), an increase of $45 \%$ in the mean value strength, from larger specimens to smaller ones, is predicted. However, experimental results show only a $9.1 \%$ increase in strength. Therefore, the absolute error in the Weibull prediction for strength change is $|45-9.1|=35.9 \%$. In the case of Japanese larch, an increase of $41.4 \%$ in the mean strength is predicted by the CWSEL. However, there is only a $7.8 \%$ increase in the strength according to experiments. In this case, the absolute error introduced in the prediction of strength change due to size effect is $|41.4-7.8|=33.6 \%$.

To highlight the inaccuracy of the classical law, the relative error is also calculated. This value shows the error in prediction of strength change compared to the absolute value of the change obtained from experimental results. The relative errors, in the case of spruce and Japanese larch, are $|45-9.1| / 9.1 \times 100=394 \%$ and $|41.4-7.8| / 7.8 \times 100=431 \%$, respectively. In both cases, the CWSEL overestimates the change in strength by a factor of 5 , approximately.

The above calculations show that the CWSEL can severely overestimate the effect of size on timber strength.

\section{Strength of timber as random field}

Random variability and spatial variability in material properties usually exist in parallel. For example, in a set of specimens prepared for standard mechanical tests, the properties randomly change from one to another. Also, these specimens have already been cut from spatially different places in a timber board or composite panel that comprises spatial variability. The combination of random and spatial variability is referred to as random spatial variability. Taking this variability into account can result in more accurate and realistic numerical models, leading to more reliable and optimized timber-based designs. However, the spatial variability in the properties of clear timber has frequently been neglected in the literature [3-12].

In traditional designs, a safety factor is applied to the characteristic value of strength to obtain the design value. This safety factor is normally chosen as being sufficiently high to guarantee the safety of structures in different working conditions. However, this safety factor is a rough value and can be too conservative [27]. On the other hand, as highlighted in a recent study of the failure analysis of unidirectional composites [28], the traditional approach can also significantly overestimate structural reliability. The accurate determination of the spatial variability effect on the stochastic response of timber structures can result in more reliable timber designs for different structures and loading conditions. Although knots are frequently present in timber structures, in some applications such as timber joints, the failure usually occurs in the clear wood that is being considered in this work. Moreover, the current study can form a basis for developing models for structures containing knots.

The stochastic/random field approach can be used to model the random spatial variation of the strength [29]. Unlike composite laminas and laminates, which can usually be safely considered as 2D structures such as plates due to their low thickness, timber structures are normally modeled as beam, columns etc., which have 3D spatial variation along their lengths 
as well as their cross sections. Hence, a 3D random field should be used for the appropriate modeling of the strength field in timber.

To model timber strength as a random field, statistical variability and spatial variability have to be analytically formulated. Statistical variability is described by the marginal distribution of the random field. Marginal distribution is the statistical distribution of samples of the random field simulated in a small volume of material in which spatial variability can be neglected. In other words, the simulated field has the same value throughout a specific sample, but this can change from one sample to another. Also, spatial variability is normally characterized using an appropriate autocorrelation function that specifies the degree of correlation in strength values at a specific material point and its surrounding points. The term auto indicates the correlation between values of the same parameter. This autocorrelation function contributes to the generation of realizations of the random field, as shown in the next section.

The autocorrelation function of the random field $Y(\mathbf{x})$ is defined by the following equation [27]:

$R_{Y Y}=\int_{-\infty}^{+\infty} \int_{-\infty}^{+\infty} y_{1} y_{2} f_{Y Y}\left(y_{1}, y_{2}\right) d y_{1} d y_{2}$

where random variables $y_{1}$ and $y_{2}$ represent $Y(\mathbf{x})$ at the positions $\mathbf{x}_{1}$ and $\mathbf{x}_{2}$, and $f_{Y Y}\left(y_{1}, y_{2}\right)$ is the joint probability density function (PDF) of the random variables $y_{1}$ and $y_{2}$ . The marginal PDF is not always available [30], and only the marginal PDF is usually at hand:

$f_{Y}\left(y_{1}\right)=\int_{-\infty}^{+\infty} f_{Y Y}\left(y_{1}, y_{2}\right) d y_{2}$

which is independent of $\mathbf{x}$ for stationary random fields, as is the case in the current study. In practice, different autocorrelation functions have been developed that can be used for different random fields [23]. Concerning material parameters, exponential and squared exponential autocorrelation functions have proved to be most successful [29,31-34]. Some applications for material characterization include unidirectional composites [29], strand chopped mats [35], concrete [36] and glass fibers [26]. The squared exponential function was selected in the present work, as it has been used more often for fibrous composites [29,31,34,35] and is expressed as:

$R=\sigma^{2} \mathrm{e}^{-\left(\xi_{1}^{2}+\xi_{2}^{2}+\xi_{3}^{2}\right) / d^{2}}$

where $\sigma$ is the standard deviation of the strength field, $d$ is a correlation length and $\xi_{1}, \xi_{2}$ and $\xi_{3}$ are separation distance components along $x_{1}, x_{2}$ and $x_{3}$ directions respectively. Correlation length specifies the level of correlation in space; the shorter the correlation length, the faster the correlation decreases. The assumption of having the same value for correlation length in the longitudinal and transverse directions is commonly found in the literature [29,31,34,35] for composites. This assumption would be more justifiable for timber structures where the cross-sectional dimensions of structural members are normally smaller than the longitudinal ones and also the ones used in this study. Nevertheless, due to the orthotropic 
behavior of timber, it is most likely that the correlation length would be different in the transverse direction, which is commonly considered to be the isotropic plane. More experimental investigations will be conducted within this project to investigate this case.

\section{Random field modeling procedure}

In order to model timber strength as a random field it is necessary to generate spatial variation of strength in a specified domain, and to obtain the desired response based on this spatial variation.

\section{Generating realizations of strength as a $3 D$ random field}

Within the framework of the spectral representation scheme, simulation of a non-Gaussian random field is based on simulating an underlying Gaussian one. After this underlying Gaussian random field has been determined, the translation technique can be applied to generate realizations of the target non-Gaussian field. The translation technique is a memoryless non-linear transformation of a Gaussian field into a non-Gaussian field [37].

The method for simulating a Gaussian random process was first presented by Shinozuka and Jan [38] and later extended to multidimensional random fields [39]. In this method, the Gaussian random field $f_{G}(\mathbf{x})$ is expanded as a sum of cosine functions having random phase angles and deterministic amplitudes. The amplitudes depend on the power spectral density function of the random field, which in $3 \mathrm{D}$ space is defined as:

$$
S_{G}(\boldsymbol{\kappa})=\frac{1}{(2 \pi)^{3}} \int_{-\infty}^{\infty} \int_{-\infty}^{\infty} \int_{-\infty}^{\infty} R_{G}(\xi) \mathrm{e}^{-i\left(\kappa_{1} \xi_{1}+\kappa_{2} \xi_{2}+\kappa_{3} \xi_{3}\right)} d \xi_{1} d \xi_{2} d \xi_{3}
$$

where $R_{G}$ is the autocorrelation function and $\kappa_{1}, \kappa_{2}$ and $\kappa_{3}$ are the corresponding wave numbers. The formula to simulate the Gaussian random field is [39]

$$
\begin{aligned}
f_{G}\left(x_{1}, x_{2}, x_{3}\right)= & \sum_{n_{1}=0}^{N_{1}-1} \sum_{n_{2}=0}^{N_{2}-1} \sum_{n_{3}=0}^{N_{3}-1}\left[A _ { n _ { 1 } n _ { 2 } n _ { 3 } } \left[\cos \left(\kappa_{1 n_{1}} x_{1}+\kappa_{2 n_{2}} x_{2}+\kappa_{3 n_{3}} x_{3}+\phi_{n_{1} n_{2} n_{3}}^{1}\right)\right.\right. \\
& +\cos \left(\kappa_{1 n_{1}} x_{1}+\kappa_{2 n_{2}} x_{2}-\kappa_{3 n_{3}} x_{3}+\phi_{n_{1} n_{2} n_{3}}^{2}\right) \\
& +\cos \left(\kappa_{1 n_{1}} x_{1}-\kappa_{2 n_{2}} x_{2}+\kappa_{3 n_{3}} x_{3}+\phi_{n_{1} n_{2} n_{3}}^{3}\right) \\
& \left.\left.+\cos \left(\kappa_{1 n_{1}} x_{1}-\kappa_{2 n_{2}} x_{2}-\kappa_{3 n_{3}} x_{3}+\phi_{n_{1} n_{2} n_{3}}^{4}\right)\right]\right]
\end{aligned}
$$

where:

$$
A_{n_{1} n_{2} n_{3}}=2 \sqrt{S_{G}\left(\kappa_{1 n_{1}}, \kappa_{2 n_{2}}, \kappa_{3 n_{3}}\right) \Delta \kappa_{1} \Delta \kappa_{2} \Delta \kappa_{3}}
$$


$\kappa_{1 n_{1}}=n_{1} \Delta \kappa_{1}, \quad \kappa_{2 n_{2}}=n_{2} \Delta \kappa_{2}, \quad \kappa_{3 n_{3}}=n_{3} \Delta \kappa_{3}$

$\Delta \kappa_{1}=\frac{\kappa_{1 u}}{N_{1}}, \quad \Delta \kappa_{2}=\frac{\kappa_{2 u}}{N_{2}}, \quad \Delta \kappa_{3}=\frac{\kappa_{3 u}}{N_{3}}$

$A_{n_{1} n_{2} \mathrm{O}}=A_{n_{1} \mathrm{On} n_{3}}=A_{0 n_{2} n_{3}}=0$ for:

$n_{1}=0,1, \ldots, N_{1}-1 ; \quad n_{2}=0,1, \ldots, N_{2}-1 ; \quad n_{3}=0,1, \ldots, N_{3}-1$

In Eq. (7), $\phi_{n_{1} n_{2} n_{3}}^{i}(i=1,2,3,4)$ are random phase angles and $\kappa_{i u}(i=1,2,3)$ are upper cut-off wave numbers. $\kappa_{i u}$ are selected as being sufficiently large to cover the whole wave range of interest. Also, a random number generator is used to produce the independent random phase angles in each sample function that are uniformly distributed in the range $[0,2 \pi]$. Moreover, the fast Fourier transform technique can be applied when calculating the series in Eq. (7) to reduce the computation time of sample function generation. This is a major advantage especially when dealing with 3D random fields. As pointed out in [15], the computational efficiency of this method is far better than the other popular method known as the KarhunenLoeve expansion.

Recently, a simple and efficient scheme has been introduced by Shields et al. [37] for the simulation of general non-Gaussian one-dimensional random processes based on simulating an underlying Gaussian process. In the present work, an extension to 3D random fields has been introduced. This scheme is based on the translation technique for estimating the nonGaussian random field:

$f_{n G}\left(x_{1}, x_{2}, x_{3}\right)=F_{n G}^{-1}\left\{F_{G}\left[f_{G}\left(x_{1}, x_{2}, x_{3}\right)\right]\right\}$

where $F_{n G}^{-1}$ is the inverse of non-Gaussian cumulative distribution function (CDF) and $F_{G}$ denotes the CDF of the underlying Gaussian random field. If the autocorrelation function of the underlying Gaussian random field is known, this transformation can always be applied. However, the autocorrelation function of the non-Gaussian field is normally available, see (Eq. (5)), instead of the autocorrelation function of the Gaussian field. $S_{n G}$ can therefore be obtained by substituting $R_{n G}$ estimated by Eq. (5) into Eq. (6):

$S_{n G}=\sigma^{2} \frac{d^{3}}{8 \pi^{1.5}} e^{-\frac{d^{2}}{4}\left(\kappa_{1}^{2}+\kappa_{2}^{2}+\kappa_{3}^{2}\right)}$

This integration was done in MATLAB. An autocorrelation function for the underlying Gaussian field is estimated in an iterative manner, using the non-Gaussian marginal distribution, and the $R_{n G}$ and $S_{n G}$ as input data. When $R_{G}$ has been estimated, a Gaussian sample function can be generated using Eq. (7). Finally, the transformation in Eq. (12) is applied to obtain the target non-Gaussian sample function, which expresses the spatial variation of the strength field in each specimen.

Random response assessment 
In this study, the Monte Carlo method was used to estimate the statistics of the stochastic response of the tensile strength of wooden specimens with different volumes. Following the above-mentioned method, realizations of the strength random field were generated in each specimen. The spatial variation of the random parameter in the generated realizations were continuous and smooth, indicating that a sufficient number of terms were considered in calculating the series in Eq. (7). The strength of a specimen under a uniform uniaxial stress state can be determined as the minimum value of the strength field in the corresponding realization, as the failure mode is brittle for timber in parallel to the grain direction. Therefore, the smoothness of the spatial distribution is important for accurate estimation of the minimum value of the field in each specimen. By generating a statistically significant number of realizations and selecting the minimum strength value in each of them, a good estimation of the statistical distribution for the strength of a specimen with a specific volume can be obtained. In the current study, a sample size of 4000 realizations has been considered for each specific volume and material property, which suffices to achieve a high level of accuracy [40]. This process is repeated for specimens with different volumes and the statistical distributions for their strengths are obtained. By comparing strength statistics, such as mean values, for specimens with different volumes, the effect of size on timber strength can be determined.

\section{Estimating random field parameters for spruce and Japanese larch}

The experimental data given in Table 1 were used for estimating the correlation length of the autocorrelation function and the marginal distribution parameters of strength as a random field for spruce and Japanese larch wood.

The two-parameter Weibull distribution has been considered to represent statistical variability. For the shape parameter, as discussed in Section 2, the mean value obtained from experimental results is used. The remaining two unknowns are the scale parameter of the marginal distribution and the correlation length. Scale parameters of two different volumes for each type of wood are shown in Table 1. Therefore, it is possible to obtain the two unknown values for each wood. By extensive numerical experimentation, assuming different values for the correlation length and scale parameter of the marginal distribution, i.e. trial and error approach, appropriate values were estimated and are given in Table 2. It can be seen that the value of the scale parameter for each wood, which has a direct relationship to the mean value, is higher than the corresponding values in Table 1. This is because the marginal mean value gives the higher bound for the strength as the volume approaches zero. In the next section, it is shown that using the values given in Table 2 a good agreement with the experimental results can be achieved.

Table 2: Parameters of marginal Weibull distribution and correlation length of strength field for spruce and Japanese larch.

\begin{tabular}{|c|c|c|c|}
\hline Type of wood & $\begin{array}{c}\text { Marginal shape } \\
\text { parameter }\end{array}$ & $\begin{array}{c}\text { Marginal scale } \\
\text { parameter }(\mathrm{MPa})\end{array}$ & $\begin{array}{c}\text { Correlation length } \\
(\mathrm{mm})\end{array}$ \\
\hline spruce & 9.3 & 155.5 & 62.0 \\
\hline Japanese larch & 4.0 & 130.9 & 225.0 \\
\hline
\end{tabular}


A value of $62 \mathrm{~mm}$ for the correlation length means that when the distance between two points inside the material is $62 \mathrm{~mm}$, the correlation between field values at these points is reduced by a factor of $\exp (-1)$ according to Eq. (5) compared to the case of two coincident points. It is noted that the field value at any specific point inside the material can be considered as a random variable, because when the coordinates of a point are substituted into Eq. (7), only statistical variability remains.

The approach used to estimate the correlation length can be considered as a reversed approach, compared to the direct approach of measuring the strength at enough number of points within a sample of the material. If the correlation length is known from a direct approach, it can be used to produce realizations of the actual strength field in specimens of different sizes and investigate the size effect. These results, are unique, provided that enough number of simulations have been performed. Therefore, the reverse procedure could also be followed; i.e., having the size effect results, a unique correlation length could be found that would reproduce those results. The addition of more specimen sets of different volumes can improve the estimation of the correlation length.

\section{Random field modeling results and discussion}

The random field modeling of longitudinal tensile strength was performed for the two volumes of spruce wood with the dimensions given in Table 1. Three indicative realizations, i.e. randomly generated spatial distribution of the strength, for both volumes are shown in Figs. 1 and 2. As can be seen, the spatial variability of strength generally exhibits a more complicated pattern for the larger volume (Fig. 2). This is because the correlation length is a material parameter that expresses the degree to which the value of the field at one specific point influences field values in the surrounding points as a function of spatial distance. Generally, the more macroscopically homogeneous a material is, the higher the value of the correlation length of its mechanical properties such as strength.
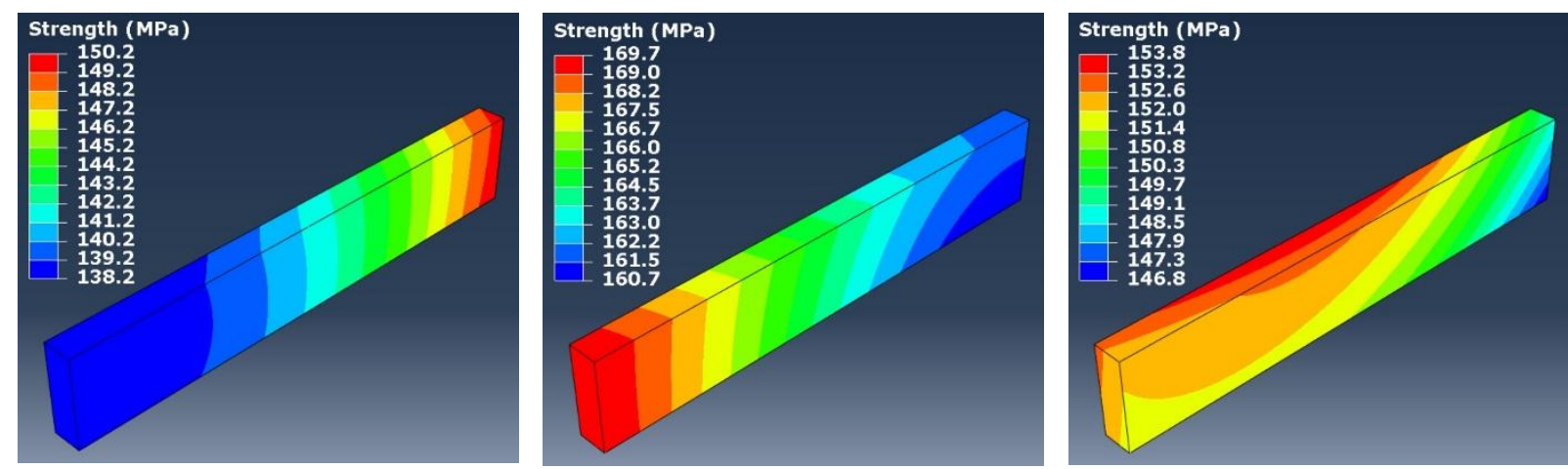

Fig. 1: Three indicative, randomly generated realizations for longitudinal tensile strength as random field in spruce specimen of volume $2 \times 6 \times 35 \mathrm{~mm}^{3}$. 

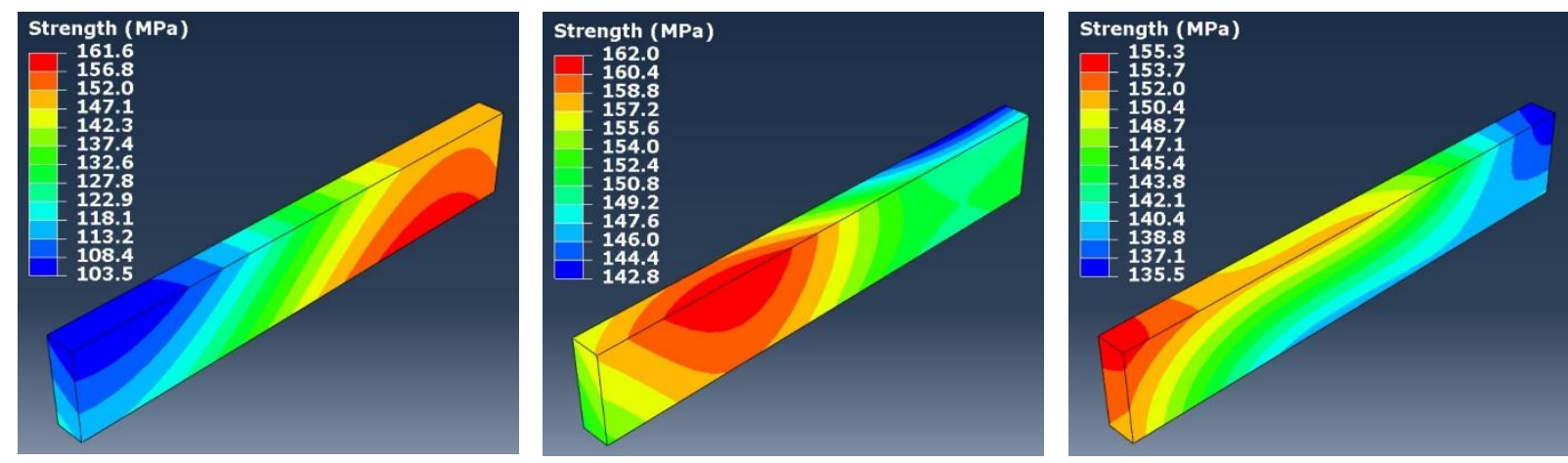

Fig. 2: Three indicative, randomly generated realizations for longitudinal tensile strength as random field in spruce specimen of volume $6 \times 20 \times 110 \mathrm{~mm}^{3}$.

As in a larger volume greater distances between material points are anticipated, the possibility of more spatial variation increases. Naturally, the probability of the occurrence of a minimum strength with a lower value also increases in each specimen, as evidenced by the numerical values of the strength field given in Figs. 1 and 2. The above constitutes the basis for the random field modeling of the size effect on strength.

Histograms of strength results from numerical simulations and fitted PDFs along with corresponding CDFs for spruce specimens with dimensions $2 \times 6 \times 35 \mathrm{~mm}^{3}$ and $6 \times 20 \times 110 \mathrm{~mm}^{3}$ are shown in Figs. 3 and 4, respectively. The three most common statistical distributions used for material properties, i.e. normal, lognormal and Weibull, have been fitted to the simulation results to ascertain which one is the most representative.
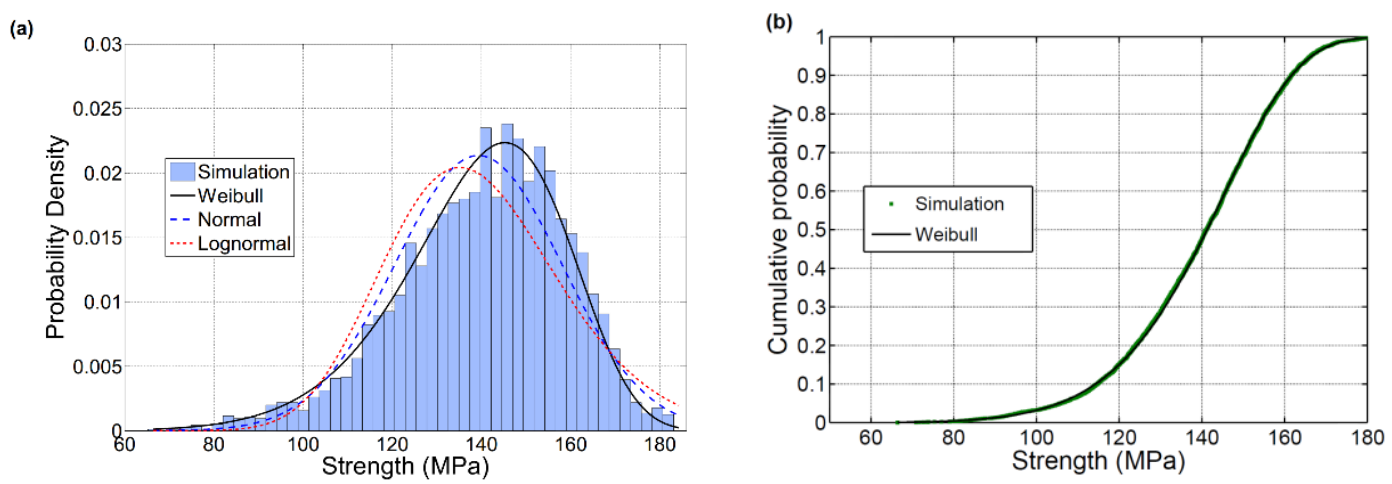

Fig. 3: a) Probability density and b) cumulative distribution functions for strength of spruce specimen with volume $2 \times 6 \times 35 \mathrm{~mm}^{3}$. 

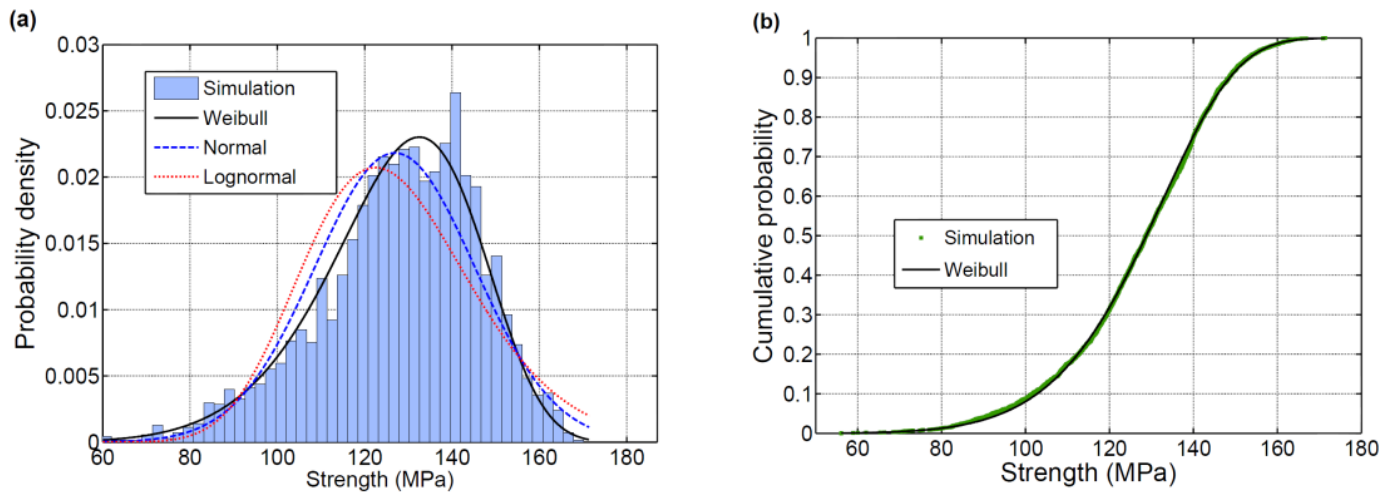

Fig. 4: a) Probability density and b) cumulative distribution functions for strength of spruce specimen with volume $6 \times 20 \times 110 \mathrm{~mm}^{3}$.

It can be seen in Figs. 3a and 4a that the Weibull PDF is the best descriptor for the simulation results. Weibull parameters as well as the results of goodness of fit tests, Anderson-Darling (AD) and Kolmogorov-Smirnov (KS), at a significance level of 0.05 are provided in Table 3. Regarding the results of the statistical tests, a value of zero denotes that sampled data are taken from the corresponding distribution, while a value of one denotes that the presumed distribution is not accepted. It is observed that both $\mathrm{AD}$ and KS tests support Weibull distribution for the longitudinal tensile strength while rejecting normal and lognormal distributions. Therefore, the strength results obtained from selecting the minimum strength value from each realization follow a Weibull distribution. Values provided for the shape parameter of Weibull distributions in Table 3 exhibit a slight change compared to that of the marginal distribution; however, as mentioned earlier, its effect on the mean value of the distribution is negligible.

Table 3: Results for statistical parameters of simulations and goodness of fit tests for longitudinal tensile strength of spruce.

\begin{tabular}{|c|c|c|c|c|c|c|c|c|}
\hline $\begin{array}{c}\text { Dimensions of } \\
\text { spruce specime } \\
\left(\mathrm{mm}^{3}\right)\end{array}$ & \multicolumn{2}{|c|}{ Weibull parameters } & \multicolumn{2}{c|}{ Weibull } & \multicolumn{2}{c|}{ Normal } & \multicolumn{2}{c|}{ Lognormal } \\
\cline { 2 - 9 } & Scale factor & Shape factor & AD & KS & AD & KS & AD & KS \\
\hline $2 \times 6 \times 35$ & 147.2 & 8.9 & 0 & 0 & 1 & 1 & 1 & 1 \\
\hline $6 \times 20 \times 110$ & 134.4 & 8.5 & 0 & 0 & 1 & 1 & 1 & 1 \\
\hline
\end{tabular}

The random field modeling of longitudinal strength was also performed for Japanese larch wood. Simulation results and fitted PDFs along with corresponding CDFs are shown in Figs. 5 and 6, respectively. Similarly to the previous case, the Weibull PDF is the best descriptor for the simulation results. 
(a)

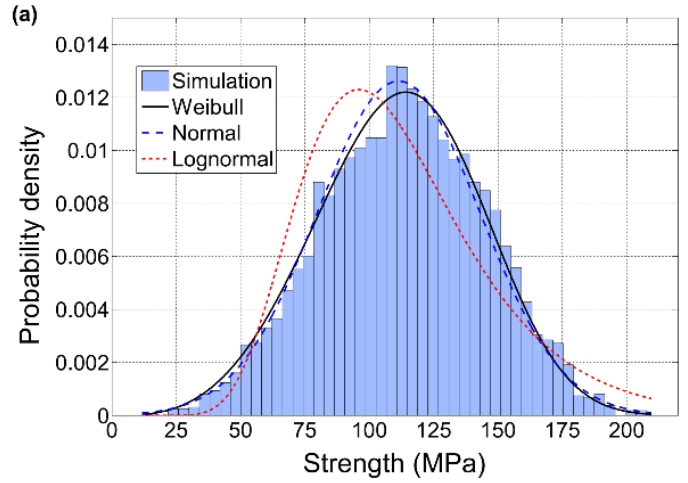

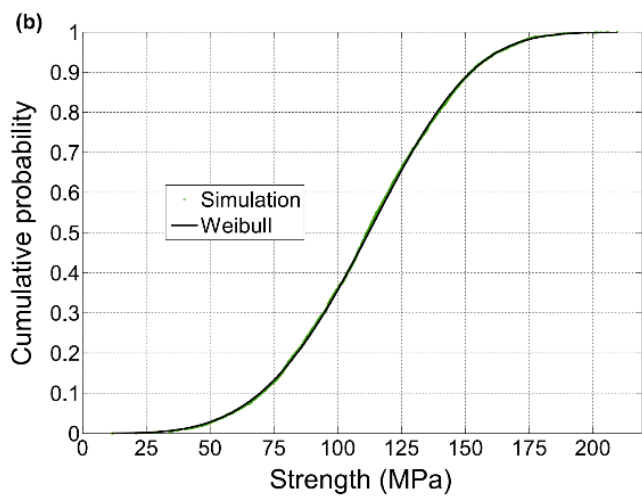

Fig. 5: a) Probability density and b) cumulative distribution functions for strength of Japanese larch specimen with volume $5 \times 25 \times 30 \mathrm{~mm}^{3}$.
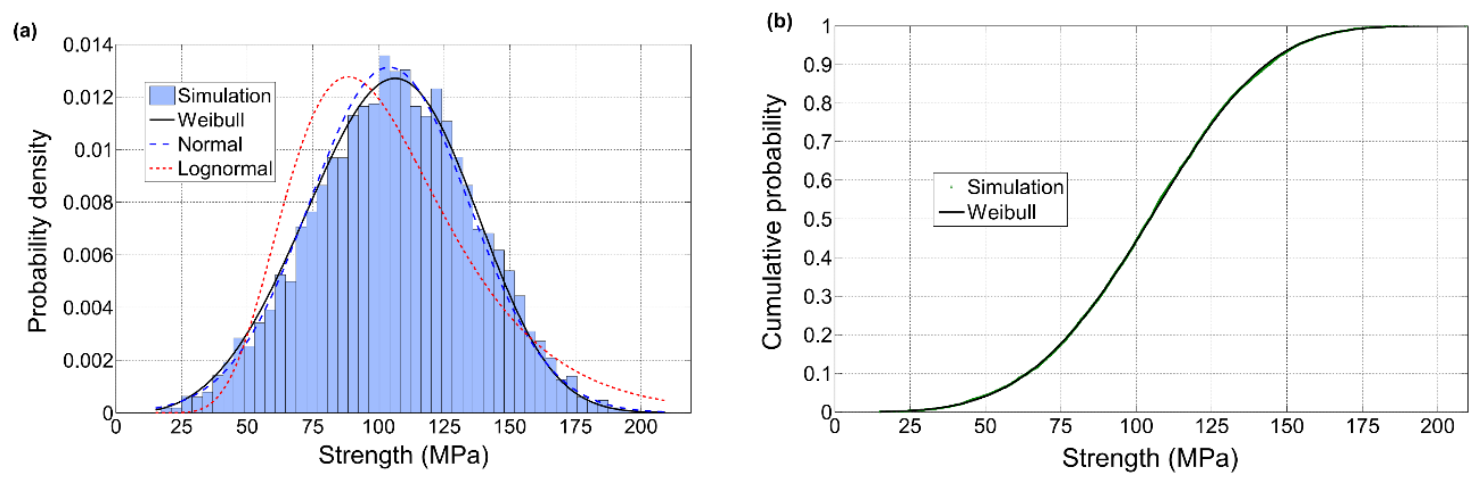

Fig. 6: a) Probability density and b) cumulative distribution functions for strength of Japanese larch specimen with volume $5 \times 25 \times 120 \mathrm{~mm}^{3}$.

Weibull parameters as well as results of goodness of fit tests are given in Table 4. Both AD and KS tests support the Weibull distribution for the longitudinal tensile strength while rejecting lognormal distribution. However, a normal distribution was only rejected by the AD test. The effect of a small variation in values for the Weibull shape parameter, given in Table 4 , on the mean values is again negligible.

Table 4: Results for statistical parameters of simulations and goodness of fit tests for longitudinal tensile strength of Japanese larch.

\begin{tabular}{|c|c|c|c|c|c|c|c|c|}
\hline $\begin{array}{c}\text { Dimensions of } \\
\text { spruce specime } \\
\left(\mathrm{mm}^{3}\right)\end{array}$ & \multicolumn{2}{|c|}{ Weibull parameters } & \multicolumn{2}{c|}{ Weibull } & \multicolumn{2}{c|}{ Normal } & \multicolumn{2}{c|}{ Lognormal } \\
\cline { 2 - 9 } & Scale factor & Shape factor & AD & KS & AD & KS & AD & KS \\
\hline $5 \times 25 \times 30$ & 123.2 & 3.9 & 0 & 0 & 1 & 0 & 1 & 1 \\
\hline $5 \times 25 \times 120$ & 115.1 & 3.8 & 0 & 0 & 1 & 0 & 1 & 1 \\
\hline
\end{tabular}

Based on the parameters in Tables 3 and 4, the prediction accuracy of the random field modeling can be evaluated by computing the ratio between the mean values of strength for shorter and longer specimens. The mean values are summarized in Table 5. This ratio is 1.098 for spruce and 1.072 for Japanese larch; i.e., a $9.8 \%$ and $7.2 \%$ increase in the strength for smaller specimens compared to the larger ones, respectively. From the experimental data, the 
corresponding values are $9.1 \%$ and $7.8 \%$. The relative errors of random field predictions compared to experiments, for both cases, are computed as $|9.8-9.1| / 9.1 \times 100=7.7 \%$ and $|7.2-7.8| / 7.8 \times 100=7.7 \%$. It can be seen that the error has been reduced from $394 \%$ and $431 \%$ to $7.7 \%$ for both woods by using the random field approach instead of the CWSEL.

Table 5: Mean values for longitudinal tensile strength of spruce and Japanese larch based on simulations.

\begin{tabular}{|l|l|l|}
\hline Type of wood & $\begin{array}{l}\text { Specimen } \\
\text { dimensions } \\
\left(\mathrm{mm}^{3}\right)\end{array}$ & $\begin{array}{l}\text { Mean value } \\
\text { strength } \\
(\mathrm{MPa})\end{array}$ \\
\hline Spruce & $2 \times 6 \times 35$ & $139.3 \pm 18.7$ \\
\cline { 2 - 3 } & $6 \times 20 \times 110$ & $126.9 \pm 17.8$ \\
\hline Japanese larch & $5 \times 25 \times 30$ & $111.5 \pm 32.0$ \\
\cline { 2 - 3 } & $5 \times 25 \times 120$ & $104.0 \pm 30.6$ \\
\hline
\end{tabular}

\section{Size effect in wider volume ranges of clear timber and analytical approximation}

The developed model can be used for investigating the size effect on strength in a wider volume range of clear timber. First, Japanese larch is considered because the size effect is examined as specimen length changes; therefore, owing to the change in only one dimension, it is easier to demonstrate the asymptotic behaviors of the size effect. Fig. 7 displays simulation results using red dots for the mean value of strength of a specimen with crosssectional dimensions $5 \times 25 \mathrm{~mm}^{2}$ versus its length on a logarithmic scale. It can be seen that there are two asymptotes. In the left asymptote where specimen length decreases to zero, the curve gradually approaches a constant value. Based on visual observations of this curve, the length limit below which the strength can be considered to be independent of specimen length is approximately 0.1 of the correlation length, $d$. The upper bound mean value for the strength of the Japanese larch specimen is $114.9 \mathrm{MPa}$, which is lower than the mean value of the marginal distribution, $118.6 \mathrm{MPa}$, calculated based on the data in Table 2 and shown as a solid horizontal line in Fig. 7. This difference highlights the fact that the strength field was modeled as a 3D random field, and therefore, strength can change spatially across the cross section, even when the length approaches zero, leading to a decrease in the mean value of strength. As the length of the specimen increases, strength variation gradually approaches a line with a constant slope similar to that of the CWSEL. This indicates that for a very long specimen, the effect of the spatial correlation between adjacent material points becomes insignificant. Therefore, the prediction by the current model approaches that of the CWSEL in which spatial correlation is neglected. The length of specimen above which the CWSEL can be used accurately is approximately 10 times the correlation length by visual observation of the curve in Fig. 7. 


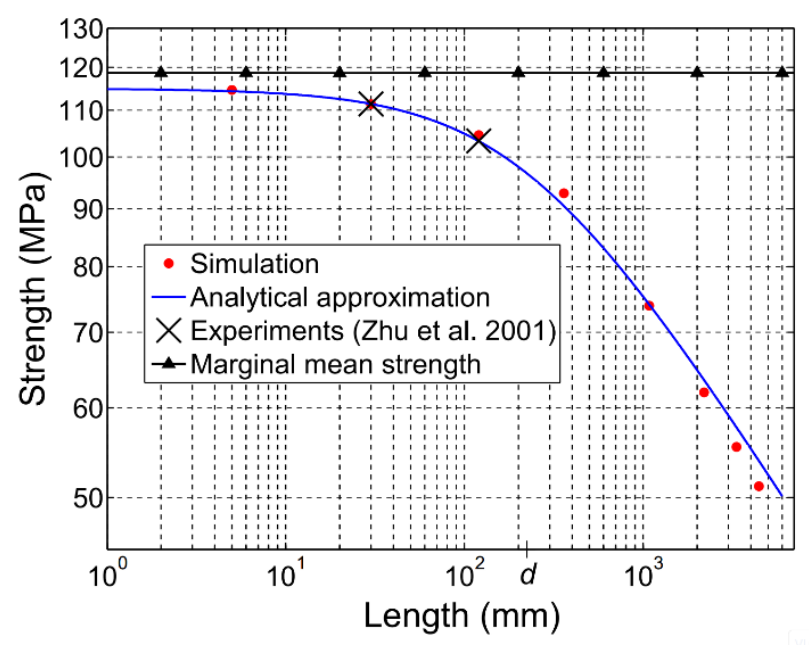

Fig. 7: Mean value strength versus length of Japanese larch specimen. $d$ on horizontal axis indicates correlation length of Japanese larch wood.

An analytical formula is proposed in the following that approximates the numerical results reasonably well and facilitates the application of the proposed size effect model for clear timber. This formula mainly modifies the part of Eq. (1) related to the volume of the material:

$$
\bar{\sigma}\left(L_{1}, L_{2}, L_{3}\right)=\sigma_{0 m} \Gamma(1+1 / m)\left[\left(\frac{d}{d+L_{1}}\right)\left(\frac{d}{d+L_{2}}\right)\left(\frac{d}{d+L_{3}}\right)\right]^{1 / m}
$$

where $L_{1}, L_{2}$ and $L_{3}$ are dimensions along $x_{1}, x_{2}$ and $x_{3}$ directions, respectively, for a specimen with uniform uniaxial stress state, and $d$ is the correlation length already introduced in Eq. (5).

Eq. (14) is proposed based on the following arguments: 1) The general form of the classical formula in Eq. (1) has been used as a basis for this equation and the shape parameter of the Weibull distribution has been used in the same manner. 2) Contrary to the CWSEL, the effect of volume change on the strength has been broken into three components corresponding to three coordinate axes, because the level of spatial variability in each direction depends on the corresponding specimen dimension. 3) The reference volume in Eq. (14) has been considered as being a very small volume of the material in which spatial variability can be neglected. Thus, when specimen dimensions approach zero, the expression powered by $1 / \mathrm{m}$ approaches one, similar to the classical formula. Naturally, what remains should represent the mean value of the marginal distribution. Therefore, $\sigma_{0}$ in Eq. (1) has been changed to $\sigma_{0 m}$ in Eq. (14).

4) The correlation length of the strength field has been incorporated in the classical formula, in such a way that the asymptotic behaviors of the size effect predicted by the numerical simulations hold.

Eq. (14) cannot be arbitrarily scaled because the correlation length, $d$, as a material parameter, introduces a length scale into the size effect law in a natural way. This equation has also been plotted in Fig. 7. The two above-mentioned asymptotic behaviors observed in the numerical results are also satisfied by this equation. In other words, the mean value of strength approaches a constant value as specimen dimensions become small compared to the correlation length. On the other hand, the CWSEL can be applied when specimen dimensions are much larger than the correlation length. Finally, it is noted that Eq. (14) also predicts a 
lower left asymptotic value of strength than the marginal strength for the Japanese larch specimen with cross-sectional dimensions $5 \times 25 \mathrm{~mm}^{2}$ because when the length of the specimen approaches zero, only $L_{3}$ is removed in Eq. (14). Therefore, the expression powered by $1 / m$ will still have a value lower than 1 .

A similar analysis can be performed for spruce wood. However, because all the specimen dimensions vary in this case, it is not possible to plot strength variations as a function of $L_{1}$, $L_{2}$ and $L_{3}$. In Table 6 , the mean value strengths of specimens with various volumes obtained from numerical simulations have been compared to those predicted by Eq. (14). The results of the analytical formula are well corroborated by the numerical simulations except for larger volumes where the error increases up to $11.2 \%$, see Table 6 . When all the specimen dimensions become very small, the predicted mean strength value approaches the marginal mean value. For large specimen dimensions, the prediction by the current model approaches that of the CWSEL.

Table 6: Comparison of numerical results for mean value of strength (MPa) of spruce wood with predicted values of analytical approximation.

\begin{tabular}{|l|l|l|l|}
\hline Dimensions (mm) & Numerical simulation & Analytical approximation & Error (\%) \\
\hline $2 \times 2 \times 2$ & 146.5 & 146.0 & 0.34 \\
\hline $2 \times 6 \times 35$ & 139.3 & 138.7 & 0.43 \\
\hline $6 \times 20 \times 110$ & 127.0 & 126.9 & 0.079 \\
\hline $9.6 \times 28.8 \times 163.2$ & 121.6 & 121.3 & 0.25 \\
\hline $19.2 \times 57.6 \times 326.4$ & 107.2 & 109.6 & 2.2 \\
\hline $57.6 \times 172.8 \times 979.2$ & 83.8 & 87.9 & 4.9 \\
\hline $172.8 \times 518.4 \times 2937.6$ & 61.3 & 66.2 & 8.0 \\
\hline $1036.8 \times 1036.8 \times 2937.6$ & 47.1 & 52.4 & 11.2 \\
\hline
\end{tabular}

\section{Conclusions}

In this paper, a random field-based size effect model has been proposed for the longitudinal tensile strength of timber. The statistical and spatial variability of the strength as a 3D random field were taken into account by its marginal distribution and autocorrelation function. Realizations of the strength field were generated in specimens with different volumes using the spectral representation scheme, and the strength results were obtained based on the Monte Carlo method along with the weakest link concept. The main conclusions are as follows:

- The application of the CWSEL to the experimental data in the literature showed a very high level of error. On the other hand, the current model was in a good agreement with the experimental data.

- The current model predicts an upper bound for the strength as specimen dimensions approach zero. On the other hand, when the dimensions are sufficiently large 
compared to the correlation length of the strength field, the result obtained by the current model approaches that of the CWSEL.

- It was shown that the limits for the left and right asymptotic behaviors of the model are approximately 0.1 and 10 times the correlation length because, in the denominator of Eq. (14), if $L$ is much smaller than $d, L$ can be disregarded and vice versa. This range covers most real applications, which emphasizes the practical aspect of the current study.

- Values of $62 \mathrm{~mm}$ and $225 \mathrm{~mm}$ for the correlation lengths of the strength fields of spruce and Japanese larch were obtained. These values can be used in the stochastic simulation of timber structures under different loading conditions such as bending. Also, this indicates that the correlation length of the strength field in timber can vary significantly between different species.

- Finally, an analytical formula was proposed that closely approximates numerical results and facilitates the application of the current model. This formula, unlike its classical counterpart, is not arbitrarily scalable and includes the correlation length as a length scale.

The proposed model is in good agreement with experimental data, and it seems more appropriate than the CWSEL, particularly in the FE context, to extend the size effect predictions to small volumes. This can improve the current design of timber structures, such as timber joints, in which the size effect on the strength of clear wood is important. In this regard, conducting additional tests are suggested with different species. This work can also constitute a preliminary step toward developing models that take knots into account.

\section{Acknowledgement}

The authors wish to acknowledge the funding of this work by the National Research Program NRP 66 of the Swiss National Science Foundation (Grant No. 406640-136680).

\section{References}

[1] Weibull, W., 1939. A statistical theory of strength of materials. In: Proceedings of the Royal Swedish Institute. Research No. 151, Stockholm, Sweden.

[2] Arwade, S.R., Clouston, P.L., Krupka, M.T., 2011. Length effects in tensile strength in the orthogonal directions of structural composite lumber. Journal of Testing and Evaluation 39, 1-7.

[3] Vallée, T., Tannert, T., Ganne-Chedville, C., 2012. Capacity prediction of welded timber joints. Wood Science and Technology 46, 333-347.

[4] Tannert, T., Vallée, T., Hehl, S., 2012. Probabilistic strength prediction of adhesively bonded timber joints. Wood Science and Technology 46, 503-513.

[5] Tannert, T., Vallée, T., Hehl, S., 2012. Experimental and numerical investigations on adhesively bonded hardwood joints. International Journal of Adhesion and Adhesives 37, 65-69.

[6] Tannert, T., Lam, F., Vallée, T., 2011. Structural performance of rounded dovetail connections: Experimental and numerical investigations. European Journal of Wood and Wood Products 69, 471482.

[7] Clouston, P.L., Lam, F., 2002. A stochastic plasticity approach to strength modeling of strandbased wood composites. Composites Science and Technology 62, 1381-1395. 
[8] Clouston, P.L., Lam, F., 2001. Computational modeling of strand-based wood composites. Journal of Engineering Mechanics 127, 844-851.

[9] Clouston, P., Lam, F., Barrett, J.D., 1998. Incorporating size effects in the Tsai-Wu strength theory for Douglas-fir laminated veneer. Wood Science and Technology 32, 215-226.

[10] Madsen, B., 1990. Size effects in defect-free Douglas fir. Canadian Journal of Civil Engineering $17,238-242$.

[11] Madsen, B., Tomoi, M., 1991. Size effects occurring in defect-free spruce-pine-fir bending specimens. Canadian journal of civil engineering 18, 637-643.

[12] Madsen, B., 1990. Length effects in $38 \mathrm{~mm}$ spruce-pine-fir dimension lumber. Canadian Journal of Civil Engineering 17, 226-237.

[13] Dill-Langer, G., Hidalgo, R.C., Kun, F., Moreno, Y., Aicher, S., Herrmann, H.J., 2003. Size dependency of tension strength in natural fiber composites. Physica A: Statistical Mechanics and its Applications 325, 547-560.

[14] Zhu, J., Kudo, A., Takeda, T., Tokumoto, M., 2001. Methods to estimate the length effect on tensile strength parallel to the grain in Japanese larch. Journal of Wood Science 47, 269-274.

[15] Stefanou, G., Papadrakakis, M., 2007. Assessment of spectral representation and Karhunen-Loève expansion methods for the simulation of Gaussian stochastic fields. Computer Methods in Applied Mechanics and Engineering 196, 2465-2477.

[16] Arwade, S.R., Clouston, P.L., Winans, R., 2009. Measurement and stochastic computational modeling of the elastic properties of parallel strand lumber. Journal of Engineering Mechanics 135, 897-905.

[17] Arwade, S.R., Winans, R., Clouston, P.L., 2010. Variability of the compressive strength of parallel strand lumber. Journal of Engineering Mechanics 136, 405-412.

[18] Gumbel, E.J., 1958. Statistics of Extremes. Columbia University Press, New York (reprinted in 2004 by Dover, New York).

[19] Adler, R.J., Taylor, J.E., 2007. Random fields and Geometry, Springer Monographs in Mathematics, Springer, New York.

[20] Katz R.W., Parlange M.B., Naveau P., 2002. Statistics of extremes in hydrology. Advances in Water Resources 25, 1287-1304.

[21] Beniston, M., Stephenson, D.B., Christensen, O.B., Ferro, C.A.T., Frei, C., Goyette, S., Halsnaes, K., Holt, T., Jylhä, K., Koffi, B., Palutikof, J., Schöll, R., Semmler, T., Woth, K., 2007. Future extreme events in European climate: an exploration of regional climate model projections. Climate Change 81, 71-95.

[22] Colombi, S., Davis, O., Devriendt, J., Prunet, S., Silk, J. Extreme value statistics of smooth Gaussian random fields. Monthly Notices of the Royal Astronomical Society 414, 2436-2445.

[23] Vanmarcke, E., 2010. Random fields: analysis and synthesis. World Scientific.

[24] Yakir, B., 2013. Extremes in Random Fields: A Theory and Its Applications. John Wiley \& Sons, Inc.

[25] Yeh, C.H., Rahman, M., 1998. Stochastic finite element methods for the seismic response of soils. International Journal for Numerical and Analytical Methods in Geomechanics 22, 819-850.

[26] Vořechovský, M., Chudoba, R., 2006. Stochastic modeling of multi-filament yarns: II. Random properties over the length and size effect. International Journal of Solids and Structures 43, 435-458. [27] Choi, S.K., Canfield, R.A., Grandhi, R.V., 2007. Reliability-based structural design, SpringerVerlag, London.

[28] Lekou D.J., Philippidis T.P., 2008. Mechanical property variability in FRP laminates and its effect on failure prediction. Composites Part B: Engineering 39, 1247-56.

[29] Wu, W.F., Cheng, H.C., Kang, C.K., 2000. Random field formulation of composite laminates. Composite Structures 49, 87-93.

[30] Sudret, B., der Kiureghian, A., 2000. Stochastic finite element methods and reliability: a state-ofthe-art report. Rep. No. UCB/SEMM-2000/08, University of California at Berkeley, USA. 
[31] Stefanou, G., Papadrakakis, M., 2004. Stochastic finite element analysis of shells with combined random material and geometric properties. Computer Methods in Applied Mechanics and Engineering 193, 139-160.

[32] Ngah, M.F., Young, A., 2007. Application of the spectral stochastic finite element method for performance prediction of composite structures. Composite Structures 78, 447-456.

[33] Chen, N.Z., Guedes Soares, C., 2008. Spectral stochastic finite element analysis for laminated composite plates. Computer Methods in Applied Mechanics and Engineering 197, 4830-4839.

[34] Sasikumar, P., Suresh, R., Gupta, S., 2013. Stochastic finite element analysis of layered composite beams with spatially varying non-Gaussian inhomogeneities. Acta Mechanica, 1-20.

[35] Sriramula, S., Chryssanthopoulos, M.K., 2013. An experimental characterisation of spatial variability in GFRP composite panels. Structural Safety 42, 1-11.

[36] Vořechovský, M., 2007. Interplay of size effects in concrete specimens under tension studied via computational stochastic fracture mechanics. International Journal of Solids and Structures 44, 2715 2731.

[37] Shields, M.D., Deodatis, G., Bocchini, P., 2011. A simple and efficient methodology to approximate a general non-Gaussian stationary stochastic process by a translation process.

Probabilistic Engineering Mechanics 26, 511-519.

[38] Shinozuka, M., Jan, C.M., 1972. Digital simulation of random processes and its applications. Journal of Sound and Vibration 25, 111-128.

[39] Shinozuka, M., Deodatis, G., 1996. Simulation of multi-dimensional Gaussian stochastic fields by spectral representation. Applied Mechanics Reviews 49, 29-53.

[40] Stefanou, G., 2009. The stochastic finite element method: Past, present and future. Computer Methods in Applied Mechanics and Engineering 198, 1031-1051. 